\title{
Identification of a Gene Encoding a Tumor-Specific Antigen that Causes Tumor Rejection
}

\author{
H. J. Stauss ${ }^{1}$. M. A. Fink, B. Starr, and H. Schreiber ${ }^{2}$
}

\section{A. Introduction}

Transplantation experiments have clearly demonstrated the existence of unique (individual) tumor-specific antigens on cancers induced by physical or chemical carcinogens. These antigens often induce a tumor-specific immune response upon immunization with a tumor which protects the host against a subsequent challenge with the same tumor, but not against a challenge with any other independently induced tumor [1]. Unique antigens were observed even when the tumors were induced with the same carcinogen in the same organ system in the same strain of mice [2]. This finding of unique tumor specificity raises questions about the mechanism by which these tumorspecific antigens are generated. The critical questions regarding such unique tumor-specific antigens are their composition, genetic origin, and possible role as target antigens for the immune system. However, the identification of tumor-specific antigens that cause tumor rejection has proven to be extremely difficult in the past. Serological probes with unique tumor specificity are difficult to obtain [3], and the serologically rec-

\footnotetext{
${ }^{1}$ Supported by a Fellowship of the National Cancer Cytology Center and a Fellowship of the Deutsche Forschungsgemeinschaft

${ }^{2}$ Supported by Grants PO1 CA-19266, RO1 CA22677 and RO1 CA-37156 from the National Cancer Institute

Department of Pathology, University of Chicago, Chicago, IL 60637, USA
}

ognized antigens may not be the target for tumor rejection [4] that is primarily $\mathrm{T}$-cell mediated [5].

We used UV-induced tumors of mice for studying the nature of tumor-specific antigens for the following reasons: (a) the unique tumor-specific rejection antigens on $U V$-induced tumors are stronger than those on chemically induced tumors, in that UVinduced tumors often regress after transplantation into normal mice even without prior immunization; (b) several of the tumor-specific rejection antigens on one such UV-induced regressor tumor, called 1591$\mathrm{RE}$, have been defined by cytolytic T-cell clones; (c) monoclonal antibodies with unique specificity for this UV-induced regressor tumor have been generated which reacted with novel MHC class I molecules on this tumor; and (d) the genes encoding the antibody-recognized novel class I molecules have been cloned and identified by transfection. We describe here the relationship between the novel MHC class I molecules encoded by the cloned genes and the rejection antigens of the 1591 tumor. Recently, we found that one of the novel 1591 class I genes encodes an antigen that causes immunological tumor rejection in normal mice [6]. Transfection of this novel class I gene into a 1591 progressor tumor variant leads to the rejection of the gene-transfected progressor tumor, demonstrating that a single gene can revert the progressive growth behavior and establish the regressor phenotype characteristic of the parental 1591-RE tumor. 


\section{B. Results}

The 1591 tumor contains three novel class I genes designated 216,166, and 149 which account for the abnormal reactivity of the tumor cells with MHC class I-specific monoclonal antibodies [7]. The gene 216 encodes an antigen that is selectively recognized by the 1591 tumor-specific antibody CP28 [8]. The molecules encoded by the genes 149 and 166 cross-react with monoclonal antibodies specific for allogeneic MHC class I antigens. Together, the three 1591 class I genes 216, 166 , and 149 can account for all the novel $\mathrm{MHC}$ class I determinants expressed by

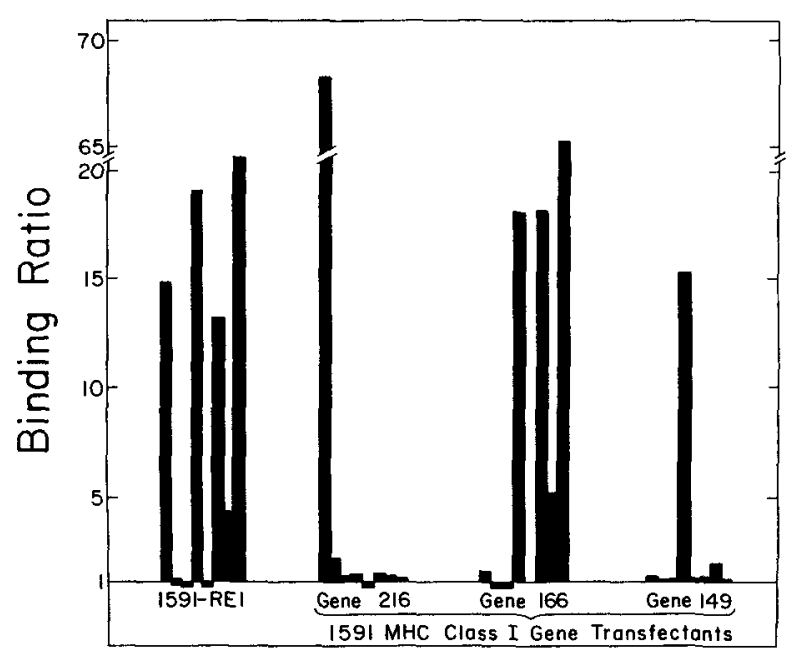

Cell Lines

Fig. 1. The 1591 class I genes 216, 166, and 149 account for all the novel MHC class I determinants that are expressed by the parental 1591-RE tumor. Furthermore, the novel class I gene 216 selectively encodes for the antigen reactive with the syngeneic tumor-specific monoclonal antibody CP28. Shown is the binding of nine MHC class Ispecific monoclonal antibodies to the parental 1591 tumor (1591-RE1) or to mouse L cells transfected with the 1591 class I genes 216,166 , and 149 , respectively. Untransfected L cells do not react with any of the nine monoclonal antibodies (not shown). The fluorescence-activated cell sorter, FACS IVB, was used to determine the indirect fluorescence listed as binding ration, i.e., fluorescence of the cells stained with the first (antiMHC class I) and second (fluoresceinated goat anti-mouse) antibody over the fluorescence of the cells stained with the second antibody alone. The bars (left to right) indicate the binding of the antibodies CP28, 34-2-12 $\left(\mathrm{D}^{\mathrm{d}}\right), 34-5-8\left(\mathrm{D}^{\mathrm{d}}\right), 28-14-8$ $\left(L^{d} D^{b}, q\right), 34-4-20\left(L^{d} D^{d}\right), 30-5-7\left(L^{d} D^{q} L^{q} L^{b}\right), 23-$ 5-21 ( $\left.\mathrm{D}^{\mathrm{b}} \mathrm{D}^{\mathrm{d}} \mathrm{D}^{\mathrm{s}}, \mathrm{q}, \mathrm{p}\right)$, and CP3F4 to the tested cells (Reproduced from Ref. 6 with permission of the editors)

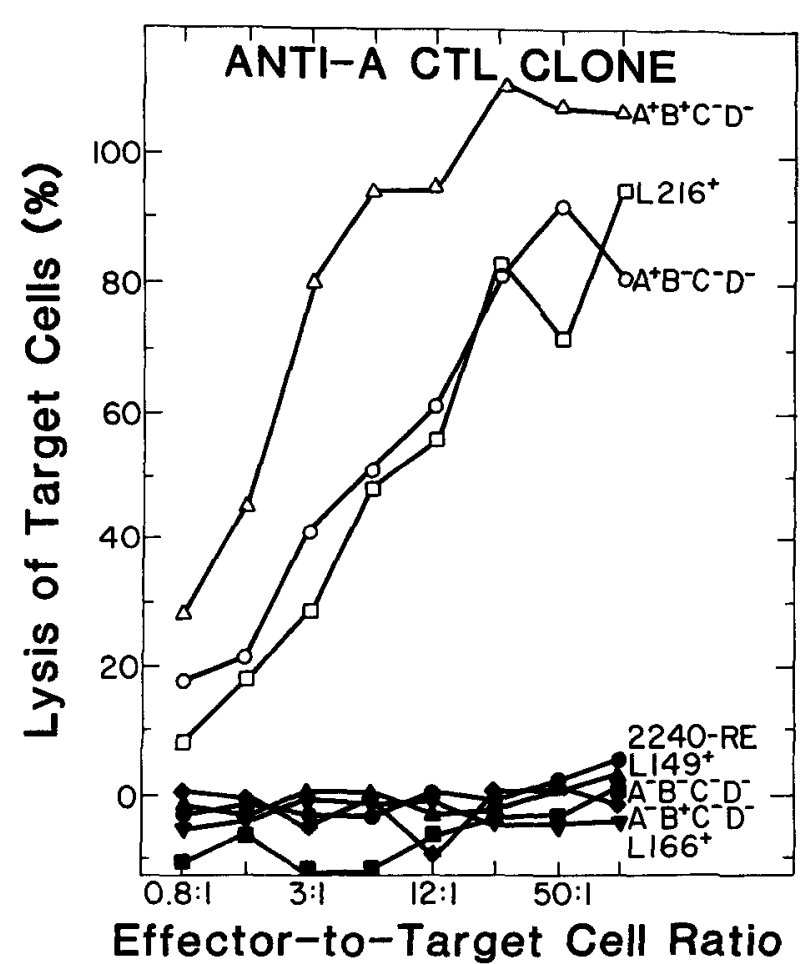

Fig. 2. The 1591 class I gene 216 encodes the antigen that is recognized by the tumor-specific anti-A CTL clone. This T-cell clone defines the A antigen on the 1591 tumor as rejection antigen because it selects in vitro from the parental regressor tumor for antigen loss variants that grow progressively in normal mice. The two 1591 regressor tumors with the phenotype $\left(\mathrm{A}^{+} \mathrm{B}^{+} \mathrm{C}^{-} \mathrm{D}^{-}\right)$and $\left(\mathrm{A}^{+}\right.$ $\mathrm{B}^{-} \mathrm{C}^{-} \mathrm{D}^{-}$), and the 216 gene-transfected $\mathrm{L}$ cells are lysed by this CTL clone. L cells transfected with the 1591 class I genes 149 or 166 or the $\mathrm{A}^{-}$ variants of the 1591 tumor or an unrelated UV-induced $\mathrm{C} 3 \mathrm{H}$ tumor (2240-RE) are not lysed in a 4.5-h ${ }^{51} \mathrm{Cr}$ release assay. $(A, B, C$, and $D$ are CTL defined 1591 tumor-specific antigens) (Reproduced from Ref. 6)

1591 tumor cells (Fig. 1). In addition, the 1591-RE1 tumor expresses multiple independent CTL-defined antigens [9] each of which can independently cause tumor rejection. In the first part of our study, we determined whether any of the three novel MHC class I genes $(216,166$, or 149) encoded the antigen recognized by anti-A CTL. We have shown previously that tumor variants selected for the loss of the anti-A CTL-defined antigen are no longer rejected by normal mice [10] implicating a close linkage between (or even identity of) the $A$ antigen and the antigen leading to tumor rejection. However, careful attempts to block A antigenspecific CTL clones with antibodies specific for any one of the three novel class I MHC 
antigens encoded by the 216,166 , or 149 genes failed [11]. Therefore, the relationship of the serologically defined novel class I antigens to the CTL-defined antigen remained uncertain. We transfected the novel 1591 class I genes into mouse L cells and used these gene-transfected cells as targets for the 1591 tumor-specific CTL lines. Figure 2 shows that only the 216 gene-transfected L cell line was killed by the anti-A CTL line while $L$ cells transfected with the 166 gene or the 149 gene were not affected by the anti-A CTL clone. The $\mathrm{A}^{-} \mathrm{B}^{+} \mathrm{C}^{-} \mathrm{D}^{-}$or

Table 1. Reversal of malignant growth in normal mice by transfection of the novel class I gene 216

\begin{tabular}{|c|c|c|c|}
\hline \multirow[t]{2}{*}{ Cell line ${ }^{\mathbf{a}}$} & \multirow{2}{*}{$\begin{array}{l}\text { Expression } \\
\text { of the } 216 \\
\text { gene } \\
\text { product }^{b}\end{array}$} & \multicolumn{2}{|c|}{ Tumor incidence $^{\mathfrak{c}}$} \\
\hline & & $\begin{array}{l}\text { Normal } \\
\text { mice }\end{array}$ & $\begin{array}{l}\text { Nude } \\
\text { mice }\end{array}$ \\
\hline $\begin{array}{l}\text { 1591-PRO } \\
\text { TR216ะ1 }\end{array}$ & + & $1 / 5^{d}$ & $2 / 2$ \\
\hline $\begin{array}{l}\text { 1591-PRO } \\
\text { TR216 } 1 \\
\text { Total }\end{array}$ & + & $\begin{array}{l}0 / 5 \\
1 / 10 \quad(10 \%)\end{array}$ & $2 / 2$ \\
\hline $\begin{array}{c}\text { 1591-PRO } \\
\text { TR216.3 }\end{array}$ & - & $5 / 5$ & $2 / 2$ \\
\hline $\begin{array}{l}\text { 1591-PRO } \\
\text { TR216-4 } \\
\text { Total }\end{array}$ & - & $\begin{array}{l}4 / 5 \\
9 / 10 \quad(90 \%)\end{array}$ & $2 / 2$ \\
\hline $\begin{array}{l}\text { 1591-PRO } \\
\text { TR216.1 } \\
\text { reisolate }^{\text {d }}\end{array}$ & - & $5 / 5 \quad(100 \%)$ & ND \\
\hline 1591-PRO & - & $8 / 10 \quad(80 \%)$ & ND \\
\hline 1591-RE & + & $0 / 10 \quad(0 \%)$ & $2 / 2$ \\
\hline
\end{tabular}

a A clone of the progressor tumor, 1591-PRO was transfected with the 216 gene and the neomycinresistant gene. The G418 drug-resistant cell population was cloned and two clones which expressed the 216 gene-encoded antigen (1591-PRO TR216:1 and 1591-PRO TR216.2) and two clones which did not express the 216 gene-encoded antigen (1591-PRO TR216.3 and 1591-PRO TR216.4) were used to challenge five normal mice or two nude mice with tumor fragments containing $>10^{8}$ tumor cells.

${ }^{b}$ Expression of the 216 gene product was determined by FACS IVB analysis using the monoclonal antibody CP28 that specifically recognized this gene product and a fluoresceinated second antibody. Cell lines designated positive for expression of the 216 gene product stained at least two times above background (binding ratio $>2$ ), while all cell lines designated negative for 216 gene the $\mathrm{A}^{-} \mathrm{B}^{-} \mathrm{C}^{-} \mathrm{D}^{-}$variants of the 1591 were not killed. As expected, however, the $\mathrm{A}^{+} \mathrm{B}^{-}$ $\mathrm{C}^{-} \mathrm{D}^{-}$variant of 1591 was killed by the antiA CTL, as was the $\mathrm{A}^{+} \mathrm{B}^{+} \mathrm{C}^{-} \mathrm{D}^{-}$parental 1591-RE regressor tumor line. Anti-B, antiC, or anti-D CTL did not kill any of the L cells transfected with the novel MHC class I genes (not shown). Together, our data clearly indicate that the 216 gene-encoded novel class I antigen is recognized by both the CP28 monoclonal antibody (Fig. 1) as well as the anti-A CTL clone (Fig. 2).

We have shown previously that all the in vivo- or in vitro-derived progressor variants of the 1591-RE tumor had lost all three novel class I genes 216, 166, and 149 simultaneously [6]. It was not clear whether the presence of the 216 gene would alone be sufficient to establish the regressor phenotype. To test this, we introduced by transfection the 216 gene into a progressively growing $\mathrm{A}^{-} 1591$ variant, designated 1591-PRO, which had lost all three novel class I genes. This progressor tumor was cotransfected with the 216 gene and the gene encoding the enzyme aminoglycoside phosphotransferase which confers resistance to the drug G418. The G418-resistant cell population was cloned and 24 of 77 clones expressed the 216 gene-encoded antigen as determined by their reactivity with the CP28 antibody. Two 216 genes expressing, $\mathrm{A}$ antigen-positive clones, designated TR216 $6^{+} .1$ and TR216 ${ }^{+} .2$, and two negative clones, designated TR216 ${ }^{-} .3$

expression stained less than 1.5 -fold above background (binding ratio $<1.5$ ).

${ }^{c}$ Number of mice with progressively growing tumors/number of mice challenged. Mice receiving the $216^{-}$clones died within approximately 6 weeks due to the large tumor burden. The mice that were challenged with the $216^{+}$transfectants did not develop tumors even after 4 months, except for one mouse that grew out an antigen loss variant approximately 2.5 weeks after injection. All cell lines used in this experiment readily formed tumors in nude mice.

d One of the mice injected with the transfected 1591-PRO TR216 1 cell line developed a progressively growing tumor that was reisolated (designated 1591-PRO TR216.1 reisolate) and reanalyzed for expression of the 216 gene by FACS IVB (Fig. 3) and for tumor incidence in normal mice. (Reproduced from Ref.6) 


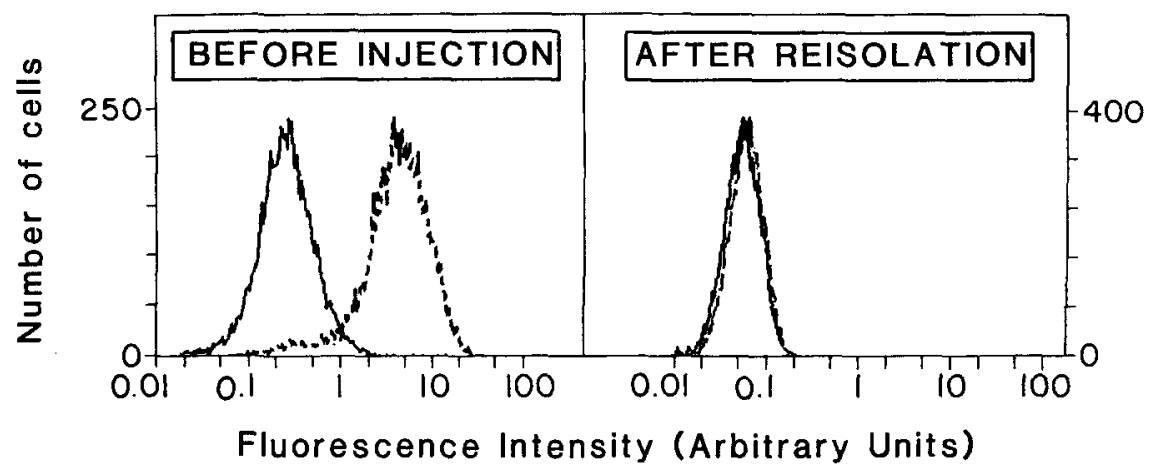

Fig. 3. Loss of expression of the transfected 216 gene in a reisolated 1591-PR0 tumor that grew progressively despite transfection with the 216 gene. The 216 gene-transfected tumors analyzed are the same as those used for the experiments described in Table 1 . The first panel shows the histogram of the 1591-PR0 TR216 ${ }^{+} .1$ cell line and the second panel shows the histogram of the 1591-PR0

and TR216 ${ }^{-} .4$, which were G418 resistant but did not express the 216 gene-encoded antigen were analyzed further. Cells of these four clones were injected into nude mice and fragments of the growing tumors were used to challenge normal animals. The use of tumor fragments grown in nude mice ensured that the cloned transfectants were still capable of growing as a malignant tumor in nude mice. Table 1 shows that the 216 gene expressing TR $216^{+} .1$ clone grew out in only one of five animals and the 216 gene expressing TR $216^{+} .2$ clone was rejected in all animals despite the fact that the mice were challenged with a large tumor dose $\left(>10^{8}\right.$ cells $)$. In contrast, the clones $\mathrm{TR} 216^{-} .3$ and TR216-.4 which do not express the 216 gene-encoded antigen grew in five of five and four of five mice, respectively, and all these mice died of progressive tumor growth. The single tumor which grew out in one of the animals that were challenged with the 216 gene expressing TR216 ${ }^{+} .1$ clone was readapted to culture and analyzed for the expression of the 216 gene-encoded antigen with the fluorescence-activated cell sorter. All cells of the reisolate were negative for the 216 antigen, indicating that the cells either lost the transfected 216 gene or that its expression was prevented by some other mechanism. This variant that did not express the 216 gene-encoded antigen was injected into five normal animals and tumor growth resulted in all the mice (Table 1). Together,
TR216.1 reisolate. Cells were incubated with the monoclonal antibody CP28 followed by incubation with fluerescein-coupled goat anti-mouse immunoglobulin antibodies (--) or incubated with only the goat anti-mouse immunoglobulin (-). Ten thousand cells were analyzed with the FACS IVB. (Reproduced from Ref. 6)

these data indicate that the stable expression of the 216 gene-encoded antigen is sufficient to change the phenotype of a progressor tumor so that it is rejected by the normal animal. Furthermore, the loss of the expression of this 216 antigen in transfected tumor cells allows these cells to regain the progressor phenotype characteristic of the untransfected parental progressor tumor.

\section{Discussion}

Many years ago, studies clearly demonstrated that tumor-specific antigens that are distinct (unique) for each individual tumor can lead to a complete immunological destruction of experimental cancers. However, the molecules eliciting (and being the target of) these immune responses have remained obscure. We have cloned and analyzed the genes encoding novel class I molecules expressed by a UV-induced murine skin tumor, designated 1591 , to determine their role in the immunobiology of tumor rejection and tumor progression. Several lines of evidence clearly indicate that one of these genes, called gene 216 , encodes an antigen that elicits 1591 tumor-specific rejection and is the target molecule of tumor rejection:

1. The 216 gene-encoded antigen must be lost before the tumor can grow progressively in a normal immunocompetent mouse. Southern blot analysis showed 
that all of the in vivo- or in vitro-derived progressor variants analyzed had lost the 216 gene [6].

2. The molecule encoded by the 216 gene is specifically recognized by the A antigenspecific cytolytic T-cell clone that we have previously shown to select in vitro for progressor variants from the parental regressor tumor cell line.

3. The most conclusive evidence comes from the fact that transfection of the 216 gene into progressively growing $1591 \mathrm{tu}-$ mor variants leads to the expression of the 216 gene-encoded antigen on the tumor and to complete rejection of all cells expressing this antigen. Thus, the progressor tumor reverted to the parental regressor phenotype following transfection.

Unique tumor-specific transplantation antigens are antigenically distinct for independently induced tumors. These different antigens may, therefore, be encoded either by numerous different unrelated genes or by a single gene which underwent multiple different mutational changes. Alternatively, these antigens might be encoded by the members of a gene family such as the immunoglobulin genes, the T-cell receptor genes, the MHC class I and class II genes, or the genes of the multiple retroviral proviruses which are present in the murine genome. Some of these gene families are known to contain the coding information for a large variety of distinct molecules and could therefore account for the observed remarkable antigenic polymorphism among tumorspecific transplantation antigens. It is interesting to notice that even a single malignant cell can express multiple unique tumor-specific antigens as has been shown for the tumor P815 [12] or 1591-RE [9]. To determine whether these antigens are encoded by a family of related genes or by multiple unrelated genes, it is necessary to analyze more tumors and to identify molecularly and genetically more unique tumor-specific transplantation antigens.

Another important and still unresolved question regarding the origin of unique tumor-specific antigens is whether the genes encoding such antigens are preexisting in the genome or whether these genes appear as the result of somatic mutation and as such re- present the product of the mutagenic action of carcinogens. Previous studies demonstrating unique antigenicity of each of the independent transformants which were all derived from one single parental cell seemed to suggest somatic carcinogen-induced mutations as a likely mechanism [13]. However, it was not excluded by these studies that the carcinogen treatment activated heritably, but at random, different preexisting, previously silent genes. Such a mechanism could also account for the observed immunogenicity of tumors in the autochthonous host [2]. In order to determine whether somatic mutations are involved in the malignant transformation and in the generation of tumor-specific antigens, we are presently searching for genetic changes in tumor cells which are not present in normal cells of the same individual.

\section{References}

1. Prehn RT, Main JM (1957) Immunity to methylcholanthrene-induced sarcomas. JNCI $18: 769$

2. Klein G, Sjogren HO, Klein E, Hellstrom KE (1960) Demonstration of resistance against methylcholanthrene-induced sarcomas in the primary autochthonous host. Cancer Res 20:1561

3. Old LJ (1982) Cancer immunology: the search for specificity. Natl Cancer Inst Monogr 60:193

4. Davey GC, Currie GA, Alexander P (1979) A serologically detected tumor-specific membrane antigen of murine lymphomas which is not the target for syngeneic graft rejection. $\mathrm{Br}$ J Cancer 40:168

5. Rouse BT, Roellinghoff $M$, Warner NL (1972) Anti-0 serum-induced suppression of cellular transfer of tumor-specific immunity to a syngeneic plasma cell tumor. Nature (New Biol) 238:116

6. Stauss HJ, Van Waes C, Fink MA, Starr B, Schreiber H (1986) Identification of a unique tumor antigen as rejection antigen by molecular cloning and gene transfer. J Exp Med 164:1516

7. Stauss HJ, Linsk R, Fischer A, Banasiak D, Haberman A, Clark I, Forman J, McMillan M, Schreiber H, Goodenow RS (1986) Isolation of the MHC genes encoding the tumorspecific class I antigens expressed on a murine fibrosarcoma. J Immunogenet 13:101-111 
8. Philipps C, McMillan M, Flood PM, Murphy DB, Forman J, Lancki D, Womack JE, Goodenow RS, Schreiber H (1985) Identification of a unique tumor-specific antigen as a novel class I major histocompatibility molecule. Proc Natl Acad Sci USA 82:5140

9. Wortzel RD, Philipps C, Schreiber H (1983) Multiple tumor-specific antigens expressed on a single tumor cell. Nature (Lond) 304:165

10. Wortzel RD, Urban JL, Schreiber H (1984) Malignant growth in the normal host after variant selection in vitro with cytolytic $T$ cell lines. Proc Natl Acad Sci USA 81:2186
11. Philipps C, Stauss HJ, Wortzel RD, Schreiber $\mathrm{H}$ (1986) A novel MHC class I molecule as tumor-specific antigen: correlation between the antibody-defined and the CTL-defined target structure. J Immunogenet 13:93

12. Uyttenhove C, Maryanski J, Boon T (1983) Escape of mouse mastocytoma P815 after nearly complete rejection is due to antigenloss variants rather than immunosuppression. J Exp Med 157:1040

13. Embleton MJ, Heidelberger C (1972) Antigenicity of clones of mouse prostate cells transformed in vitro. Int $\mathrm{J}$ Cancer 9:8 